\title{
COVARIANTLY QUANTIZED SPINNING PARTICLE AND ITS POSSIBLE CONNECTION TO NON-COMMUTATIVE SPACE-TIME
}

\author{
Subir Ghosh \\ Physics and Applied Mathematics Unit, \\ Indian Statistical Institute, \\ 203 B. T. Road, Calcutta 700108, \\ India.
}

\begin{abstract}
:
Covariant quantization of the Nambu-Goto spinning particle in 2+1-dimensions is studied. The model is relevant in the context of recent activities in non-commutative space-time. From a technical point of view also covariant quantization of the model poses an interesting problem: the set of second class constraints (in the Dirac classification scheme) is reducible. The reducibility problem is analyzed from two contrasting approaches: (i) the auxiliary variable method [8] and (ii) the projection operator method [9]. Finally in the former scheme, a Batalin-Tyutin quantization has been done. This induces a mapping between the non-commutative and the ordinary space-time. BRST quantization programme in the latter scheme has also been discussed.
\end{abstract}

Keywords: constraints, non-commutative space-time, Batalin-Tyutin quantization, spinning particle.

PACS Numbers: 02.40.Gh , 11.10Ef, 11.90.+t , .05.30.Pr 
The recent activity in Non-Commutative (NC) field theory [1] and more generally concerning $\mathrm{NC}$ space-time [2] has recreated a lot of interest in the study of physically motivated models, where the $\mathrm{NC}$ feature appears naturally. The well-known Landau problem, (of a charged particle confined in a plane in the presence of a magnetic field in the perpendicular direction), is one such example. In an earlier paper [3] we have pointed out that the bosonic Spinning

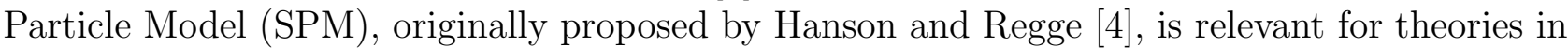
NC space-time. As we shall show in detail, (this was also pointed out briefly in [3]), the SPM can provide a direct mapping between ordinary (that is commuting) and $\mathrm{NC}$ space-time.

The Nambu Goto construction of the SPM [国], by itself, is an interesting example of a relativistic theory having a non-linear and non-abelian constraint structure. An added feature is that the system of Second Class Constraints [5] is reducible in nature if manifest Lorentz covariance is to be maintained. The present work focuses on the last point since some of the corresponding results in a non-covariant setup have already been presented by us in [3]. Quite obviously the non-covariant results are somewhat inelegant and will be difficult to use in a relativistic theory. We will discuss the preliminary steps leading towards a BRST quantization of the SPM in a manifestly covariant way, along the lines of [6, 7].

According to the Dirac classification scheme of Hamiltonian analysis of a constraint system [5], the SPM has both First Class Constraints (FCC) and a reducible system of Second Class Constraints (SCC). The former generate gauge invariance whereas the latter restrict the phase space manifold along with a modification in the canonical symplectic structure. Reducibility in a non-linear SCC system is a novel feature and possibly the present work is the first example where a nonlinear reducible SCC system is quantized. The reducibility problem for the SCCs of the SPM in a covariant framework has to be addressed first before one can embark upon a conventional BRST quantization of a set of reducible FCCs. The problem of reducibility will be handled by two very distinct approaches, i.e. the Auxiliary Variable method [B] and the Projection Operator method [9].

The BRST programme [6, 7], in Auxiliary Variable method [8], proceeds in three stages: In stage (i) the reducibility in the SCC system is taken care of by introducing a set of auxiliary degrees of freedom. This enlargement of the phase space modifies the original reducible SCCs and converts them in to an irreducible (or independent) set. However, care should be taken to see that the extension does not affect the physical, (i.e. original), phase space. In stage (ii), the set of irreducible SCCs are further modified by bringing in the Batalin-Tyutin [7] variables so that the SCCs are transformed to FCCs. Subsequently in stage (iii), the conventional BRST quantization is to be performed. Note that no ghost for ghosts appear here, (as is customary in any reducible theory), since the reducibility is removed in stage (i).

In the Projection Operator formalism [9], the reducibility problem is solved by the construction of a projection operator which projects out a maximal set of weakly involutive constraints, i.e. FCCs, (from the SCC system), with which a generalization of the standard BRST quantization is possible. In this scheme, ghost for ghosts do appear. It might be interesting to see if the auxiliary variables of the former method and secondary ghosts of the latter method are related. Indeed, the inherent nonlinearity and non-abelian nature of the SPM constraint system is a real test of the viability of the above schemes [8, 9] in arbitrary models.

The connection between SPM and NC space-time is discussed here in the auxiliary variable method. After the second stage of extension of the phase space, (where the BT variables appear), we demonstrate the existence of a mapping between the $\mathrm{NC}$ space-time coordinate 
and the normal one, via the auxiliary BT degrees of freedom [7]. The analogous results in a manifestly non-covariant setup were derived in [3]. Following the BT prescription [7] in the SPM, the NC space-time coordinate operators are expressible as normal space-time coordinates, appropriately extended by BT contributions. This provides the mapping between the NC and ordinary space-times in the extended phase space. As has been noticed in earlier studies of nonlinear theories [10, 3], the possibility of an infinite number of higher order Batalin-Tyutin (BT) variable contributions in some of the physically relevant operators manifests here also.

The paper is organized as follows. In section II a brief resume' of the SPM is provided, which also helps us to fix the notations. Section III deals with the application of the Auxiliary Variable method in SPM. In section IV, the relevant formulas for the BT quantization are provided and subsequently the method is applied to the irreducible set of SCCs in SPM, obtained in the previous section. The connection with the NC space-time is also elucidated here. In section $\mathbf{V}$ the Projection Operator in the context of SPM is derived. Necessary steps for the subsequent BRST quantization in the present case has been provided. Determination of the explicit structure of the Projection operator in a complex model is very important since its existence was only suggested in [9]. Sections III-V comprise the main body of the work. The paper is concluded with a discussion in section VI.

\section{SPINNING PARTICLE MODEL: A BRIEF RESUME'}

The 3+1-dimensional Nambu-Goto Lagrangian of the SPM, originally proposed by Hanson and Regge [4] is,

$$
L=\left[M^{2} a_{1}+\frac{J^{2}}{2} a_{2}+2 M J\left(\frac{1}{2} a_{1} a_{2}+a_{3}\right)^{\frac{1}{2}}\right]^{\frac{1}{2}} .
$$

The notations of [4] are used, where

$$
u^{\mu}=\frac{d x^{\mu}}{d \tau} \quad, \quad \sigma^{\mu \nu}=\Lambda_{\rho}^{\mu} \frac{d \Lambda^{\rho \nu}}{d \tau}=-\sigma^{\nu \mu}
$$

and the dynamical variables entering $L$ are

$$
a_{1}=u^{\mu} u_{\mu}, a_{2}=\sigma^{\mu \nu} \sigma_{\mu \nu}, a_{3}=u_{\mu} \sigma^{\mu \nu} \sigma_{\nu \lambda} u^{\lambda}, a_{4}=\operatorname{det} \sigma=\frac{1}{16}\left(\sigma^{\mu \nu} \sigma_{\mu \nu}^{*}\right)^{2}, \sigma_{\mu \nu}^{*}=\frac{1}{2} \epsilon_{\mu \nu \rho \lambda} \sigma^{\rho \lambda} .
$$

Here $\left(x^{\mu}, \Lambda^{\mu \nu}\right)$ is a Poincare group element and also a set of dynamical variables of the theory, with

$$
\Lambda_{\rho}^{\mu} \Lambda^{\rho \nu}=\Lambda_{\rho}^{\mu} \Lambda^{\nu \rho}=g^{\mu \nu} \quad, \quad g^{00}=-g^{i i}=1 .
$$

In order to discuss the Hamiltonian formulation, we define the canonically conjugate momenta as,

$$
\begin{gathered}
P^{\mu} \equiv \frac{\partial L}{\partial u_{\mu}}=L^{-1}\left[M^{2} u^{\mu}+\frac{M J}{\left(\frac{a_{1} a_{2}}{2}+a_{3}\right)^{\frac{1}{2}}}\left(\frac{1}{2} a_{2} u^{\mu}+\sigma^{\mu \nu} \sigma_{\nu \lambda} u^{\lambda}\right)\right], \\
S^{\mu \nu} \equiv \frac{\partial L}{\partial \sigma_{\mu \nu}}=L^{-1}\left[J^{2} \sigma^{\mu \nu}+\frac{M J}{\left(\frac{a_{1} a_{2}}{2}+a_{3}\right)^{\frac{1}{2}}}\left(a_{1} \sigma^{\mu \nu}+\left(u^{\mu} \sigma^{\nu \lambda}-u^{\nu} \sigma^{\mu \lambda}\right) u_{\lambda}\right)\right] .
\end{gathered}
$$

One immediately finds the primary constraints,

$$
P^{\mu} P_{\mu}=M^{2}\left(1+\frac{J^{2}}{L^{2}} \frac{a_{1} a_{4}}{\left(\frac{a_{1} a_{2}}{2}+a_{3}\right)}\right) \quad, \quad S^{\mu \nu} S_{\mu \nu}=2 J^{2} \quad, \quad S^{\mu \nu} P_{\nu}=0 .
$$


This particle model is somewhat unconventional because of the operator valued "mass" which can only reduce to the standard form for $a_{4} \approx 0$. However, we have shown [11, 3] that in $2+1$-dimensions, this complication can be avoided with the Lagrangian posited by us,

$$
L=\left[M^{2} a_{1}+\frac{J^{2}}{2} a_{2}+M J \epsilon^{\mu \nu \lambda} u_{\mu} \sigma_{\nu \lambda}\right]^{\frac{1}{2}} .
$$

With the conjugate momenta,

$$
\begin{aligned}
P^{\mu} & =\frac{\partial L}{\partial u_{\mu}}=L^{-1}\left[M^{2} u^{\mu}+\frac{M J}{2} \epsilon^{\mu \nu \lambda} \sigma_{\nu \lambda}\right] \\
S^{\mu \nu} & =\frac{\partial L}{\partial \sigma_{\mu \nu}}=\frac{L^{-1}}{2}\left[J^{2} \sigma^{\mu \nu}+M J \epsilon^{\mu \nu \lambda} u_{\lambda}\right]
\end{aligned}
$$

we obtain the constraints as,

$$
\begin{gathered}
P^{\mu} P_{\mu}=M^{2}, \quad S^{\mu \nu} S_{\mu \nu}=2 J^{2}, \\
S^{\mu \nu} P_{\nu}=0 .
\end{gathered}
$$

(9) constitutes the Casimir operators. This model has been successfully used [11, 3] in the context of anyons, i.e. excitations in 2+1-dimensions, having arbitrary spin and statistics [12]. Since the NC feature of the resulting space-time coordinates is also preserved in 2+1-dimensions from now on we will work in 2+1-dimensions. An additional set of constraints are introduced, (for a detailed discussion see [4, 11]) and the full set of constraints are,

$$
\begin{gathered}
\Psi_{1} \equiv P^{\mu} P_{\mu}-M^{2}, \Psi_{2} \equiv S^{\mu \nu} S_{\mu \nu}-2 J^{2}, \\
\Theta_{1}^{\mu} \equiv S^{\mu \nu} P_{\nu} \quad, \quad \Theta_{2}^{\mu} \equiv \Lambda^{0 \mu}-\frac{P^{\mu}}{M} \quad, \quad \mu=0,1,2 .
\end{gathered}
$$

I With the help of the following canonical Poisson Brackets (PB),

$$
\begin{gathered}
\left\{P^{\mu}, x^{\nu}\right\}=g^{\mu \nu}, \quad\left\{P^{\mu}, P^{\nu}\right\}=0 \quad, \quad\left\{x^{\mu}, x^{\nu}\right\}=0 \\
\left\{S^{\mu \nu}, S^{\lambda \sigma}\right\}=S^{\mu \lambda} g^{\nu \sigma}-S^{\mu \sigma} g^{\nu \lambda}+S^{\nu \sigma} g^{\mu \lambda}-S^{\nu \lambda} g^{\mu \sigma}, \\
\left\{\Lambda^{0 \mu}, S^{\nu \sigma}\right\}=\Lambda^{0 \nu} g^{\mu \sigma}-\Lambda^{0 \sigma} g^{\mu \nu}, \quad\left\{\Lambda^{0 \mu}, \Lambda^{0 \nu}\right\}=0
\end{gathered}
$$

we compute the constraint algebra, where $\Psi_{1}$ trivially commutes with all the constraints and the rest of the non-zero PBs between the constraints are,

$$
\begin{gathered}
\left\{\Psi_{2}, \Theta_{2}^{\mu}\right\}=4\left(S^{\mu \lambda} \Theta_{2 \lambda}+\frac{1}{M} \Theta_{1}^{\mu}\right) \quad, \quad\left\{\Psi_{2}, \Theta_{1}^{\mu}\right\}=0 \\
\left\{\Theta_{\alpha}^{\mu}, \Theta_{\beta}^{\nu}\right\} \equiv \Delta_{\alpha \beta}^{\mu \nu} \quad, \quad \alpha, \beta=1,2
\end{gathered}
$$

where,

$$
\Delta_{12}^{\mu \nu} \equiv\left\{\Theta_{1}^{\mu}, \Theta_{2}^{\nu}\right\}=\frac{1}{M}\left(M^{2} g^{\mu \nu}-P^{\mu} P^{\nu}\right)-P^{\nu} \Theta_{2}^{\mu}+g^{\mu \nu}\left(P . \Theta_{2}\right)+\frac{1}{M} g^{\mu \nu} \Psi_{1}
$$

\footnotetext{
${ }^{1}$ Note that instead of $\Psi_{2}$ as above, one can equivalently use $\Psi_{2} \equiv \epsilon^{\mu \nu \lambda} S_{\mu \nu} P_{\lambda}-M J$, which incidentally defines the Pauli Lubanski scalar.
} 


$$
\Delta_{22}^{\mu \nu} \equiv\left\{\Theta_{2}^{\mu}, \Theta_{2}^{\nu}\right\}=0, \Delta_{11}^{\mu \nu} \equiv\left\{\Theta_{1}^{\mu}, \Theta_{1}^{\nu}\right\}=M^{2} S^{\mu \nu}+\Psi_{1} S^{\mu \nu}+P^{\mu} \Theta_{1}^{\nu}-P^{\nu} \Theta_{1}^{\mu} .
$$

One can see that the constraint algebra for $\Psi_{\alpha},(\alpha=1,2)$, closes whereas, $\Delta_{\alpha \beta}^{\mu \nu}$ being nontrivial even on the constraint surface, indicates the presence of SCCs. Hence, according to the Dirac classification scheme [5], $\Psi_{\alpha}$ and $\Theta_{\alpha}^{\mu}$ constitute FCCs and SCCs respectively. Demanding time persistence of the FCCs will generate no further constraints since the theory being reparametrization invariant, its Hamiltonian will be a combination of FCCs only.

It is not possible to compute the Dirac Brackets (DB) [0] from the SCCs since the system of SCC is reducible (i.e. not independent) due to the following identity,

$$
P_{\mu} \Theta_{1}^{\mu}=0 .
$$

Note also the presence of the relation,

$$
\left(\Lambda_{\mu}^{0}+\frac{P_{\mu}}{M}\right) \Theta_{2}^{\mu}=-\frac{\Psi_{1}}{M^{2}} .
$$

However, since (19) involves an FCC, this is not a reducibility condition 13 and only restricts the number of independent degrees of freedom on the constraint manifold. Also, this system is first stage reducible since higher order reducibility conditions are absent.

The Hamiltonian of the system turns out to be that of a free particle due to the so called "rigidity" property of the particle [14, 11] meaning that when the SCCs are enforced strongly, the spin vector becomes proportional to the momentum vector. As stated before, the Hamiltonian being a combination of the FCCs (9), due to the above reason, it is sufficient to consider only the mass shell condition $\Psi_{1}$ in (9). One has to fix the time scale by choosing a gauge for the FCC $\Psi_{1}$, which can simply be

$$
x_{0}=\tau .
$$

Subsequently one has to construct the DBs for the above SCC pair and the Hamiltonian is obtained from $\Psi_{1}=0$,

$$
H \equiv P_{0}=\sqrt{P_{i} P_{i}+M^{2}} .
$$

Indeed, one can obtain the DBs by considering an irreducible set from $\Theta_{\alpha}^{\mu}$, (e.g. taking only the spatial components $\Theta_{\alpha}^{i}$, as in [3]), but this destroys the manifest covariance of the model. We now follow the method prescribed by Banerjee and Neto [8] to obtain the DBs without losing manifest covariance.

\section{COVARIANT QUANTIZATION: AUXILIARY FIELD METHOD}

In the formalism proposed in $[\mathbb{8}]$, the reducible SCCs $\left(\Theta_{\alpha}^{\mu}\right)$ are modified in an appropriate way by introducing auxiliary degrees of freedom, such that the modified SCCs $\left(\bar{\Theta}_{\alpha}^{\mu}\right)$ become irreducible. At the same time, one has to ensure that the extension does not affect the physical phase space. In a practical sense, this means that the resulting DBs between the physical (i.e. original) degrees of freedom will have to be independent of the auxiliary variables or any parameters connected to them. The reducibility condition plays a crucial role in determining the structures of the modification terms (in the SCCs), which have to be such that on imposition of the reducibility conditions on the SCCs, the auxiliary variables vanish exactly. 
The phase space is extended by introducing a canonical pair of auxiliary variables $\phi$ and $\pi$ that satisfy $\{\phi, \pi\}=1$ and PB commute with the rest of the physical variables. The SCCs $\Theta_{\alpha}^{\mu}$ are modified in the way as shown below,

$$
\bar{\Theta}_{1}^{\mu} \equiv S^{\mu \nu} P_{\nu}+k_{1} P^{\mu} \pi \quad ; \quad \bar{\Theta}_{2}^{\mu} \equiv\left(\Lambda^{0 \mu}-\frac{P^{\mu}}{M}\right)+k_{2}\left(\Lambda^{0 \mu}+\frac{P^{\mu}}{M}\right) \phi,
$$

where $k_{1}$ and $k_{2}$ denote two arbitrary parameters. The constraint matrix is computed to be,

$$
\bar{\Delta}_{\alpha \beta}^{\mu \nu} \equiv\left\{\bar{\Theta}_{\alpha}^{\mu}, \bar{\Theta}_{\beta}^{\nu}\right\}=\left(\begin{array}{cc}
\bar{\Delta}_{11}^{\mu \nu} & \bar{\Delta}_{12}^{\mu \nu} \\
-\bar{\Delta}_{12}^{\nu \mu} & \bar{\Delta}_{22}^{\mu \nu}
\end{array}\right)
$$

where,

$$
\begin{gathered}
\bar{\Delta}_{11}^{\mu \nu} \equiv\left\{\bar{\Theta}_{1}^{\mu}, \bar{\Theta}_{1}^{\nu}\right\}=\Delta_{11}^{\mu \nu}=M^{2} S^{\mu \nu}+\Psi_{1} S^{\mu \nu}+P^{\mu} S^{\nu \lambda} P_{\lambda}-P^{\nu} S^{\mu \lambda} P_{\lambda} \\
\bar{\Delta}_{21}^{\mu \nu} \equiv\left\{\bar{\Theta}_{2}^{\mu}, \bar{\Theta}_{1}^{\nu}\right\}=\frac{1}{M}\left[r\left(1+k_{2} \phi\right)+k_{1} k_{2}(1+r)\right] P^{\mu} P^{\nu}-M r\left(1+k_{2} \phi\right) g^{\mu \nu} \\
\equiv r_{1} P^{\mu} P^{\nu}+r_{2} g^{\mu \nu}, \\
\bar{\Delta}_{22}^{\mu \nu} \equiv\left\{\bar{\Theta}_{2}^{\mu}, \bar{\Theta}_{2}^{\nu}\right\}=0,
\end{gathered}
$$

with $r_{1}$ and $r_{2}$ given by,

$$
r_{1}=\frac{1-k_{2}^{2} \phi^{2}+2 k_{1} k_{2}}{M\left(1+k_{2} \phi\right)}, r_{2}=-M\left(1-k_{2} \phi\right) .
$$

In the above, we have used

$$
\Lambda^{0 \mu}=-\frac{\left(k_{2} \phi-1\right) P^{\mu}}{\left(k_{2} \phi+1\right) M} \equiv \frac{r}{M} P^{\mu},
$$

which follows directly from $\bar{\Theta}_{2}^{\mu}=0$.

Let the inverse of $\bar{\Delta}_{\alpha \beta}^{\mu \nu}$ be defined as,

$$
\bar{\Delta}_{\alpha \beta}^{\mu \nu} \bar{\Delta}_{\nu \lambda}^{\beta \gamma}=\delta_{\lambda}^{\mu} \delta_{\alpha}^{\gamma}
$$

and we consider a general form of the inverse matrix to be,

$$
\bar{\Delta}_{\nu \lambda}^{\alpha \beta}=\left(\begin{array}{cc}
0 & -a_{\nu \lambda} \\
a_{\nu \lambda} & b_{\nu \lambda}
\end{array}\right)
$$

with the entries,

$$
a_{\nu \lambda} \equiv a_{1} P_{\nu} P_{\lambda}+a_{2} g_{\nu \lambda} \quad, \quad b_{\nu \lambda} \equiv b\left(\bar{\Delta}_{11}\right)_{\nu \lambda} .
$$

The parameters $a_{1}, a_{2}$ and $b$ are found to be,

$$
a_{1}=-\frac{1-k_{2}^{2} \phi^{2}+2 k_{1} k_{2}}{2 M^{3} k_{1} k_{2}\left(1-k_{2} \phi\right)}, \quad a_{2}=-\frac{1}{r_{2}} \quad, \quad b=\frac{1}{r_{2}^{2}} .
$$


The DBs [5] are now calculated for any two generic variables $A$ and $B$ from the defining equation,

$$
\{A, B\}_{D B}=\{A, B\}-\left\{A, \bar{\Theta}_{\alpha}^{\mu}\right\} \bar{\Delta}_{\mu \nu}^{\alpha \beta}\left\{\bar{\Theta}_{\beta}^{\nu}, B\right\},
$$

where $\bar{\Delta}_{\nu \lambda}^{\alpha \beta}$ is defined in (25).

After a long and quite involved algebra we recover the DBs of the original (physical) variables in a covariant form,

$$
\begin{gathered}
\left\{x^{\mu}, x^{\nu}\right\}_{D B}=-\frac{S^{\mu \nu}}{M^{2}},\left\{P^{\mu}, x^{\nu}\right\}_{D B}=g^{\mu \nu},\left\{P^{\mu}, P^{\nu}\right\}_{D B}=\left\{P^{\mu}, \Lambda^{\nu \lambda}\right\}_{D B}=\left\{P^{\mu}, S^{\nu \lambda}\right\}_{D B}=0 \\
\left\{x^{\mu}, \Lambda^{\sigma \nu}\right\}_{D B}=\frac{1}{M^{2}} \Lambda^{\sigma \alpha}\left(g_{\alpha}^{\mu} P^{\nu}-g^{\mu \nu} P_{\alpha}\right), \\
\left\{x^{\mu}, S^{\sigma \nu}\right\}_{D B}=-\frac{1}{M^{2}}\left(S^{\mu \sigma} P^{\nu}-S^{\mu \nu} P^{\sigma}\right), \\
\left\{S^{\mu \nu}, S^{\lambda \sigma}\right\}_{D B}=S^{\mu \lambda}\left(g^{\nu \sigma}-\frac{P^{\nu} P^{\sigma}}{M^{2}}\right)-S^{\mu \sigma}\left(g^{\nu \lambda}-\frac{P^{\nu} P^{\lambda}}{M^{2}}\right)+S^{\nu \sigma}\left(g^{\mu \lambda}-\frac{P^{\nu} P^{\lambda}}{M^{2}}\right)-S^{\nu \lambda}\left(g^{\mu \sigma}-\frac{P^{\mu} P^{\sigma}}{M^{2}}\right), \\
\left\{\Lambda^{\alpha \mu}, S^{\nu \sigma}\right\}_{D B}=\Lambda^{\alpha \nu}\left(g^{\mu \sigma}-\frac{P^{\mu} P^{\sigma}}{M^{2}}\right)-\Lambda^{\alpha \sigma}\left(g^{\mu \nu}-\frac{P^{\nu} P^{\nu}}{M^{2}}\right)+\frac{\Lambda^{\alpha \beta} P_{\beta}}{M^{2}}\left(P^{\sigma} g^{\mu \nu}-P^{\nu} g^{\mu \sigma}\right) \\
\left\{\Lambda^{\alpha \mu}, \Lambda^{\beta \nu}\right\}=0 .
\end{gathered}
$$

It is very important to note that the DBs between the physical degrees of freedom are totally independent of $k_{1}$ and $k_{2}$, the parameters that appeared in conjunction with the auxiliary variables $\phi$ and $\pi$. There is no need to take a vanishing limit of $k_{1}$ and $k_{2}$. This feature ensures that the phase space extension has not disturbed the sector of physical variables. The importance of this has been repeatedly stressed in [8].

Notice that the DBs involving $P^{\mu}$ remain unchanged from the PBs but the non-commutativity in configuration space is reflected in the non-zero $\left\{x^{\mu}, x^{\nu}\right\}_{D B}$.

Quite naturally, the DBs constituting an auxiliary variable, such as the one given below,

$$
\left\{x^{\mu}, \phi\right\}_{D B}=\frac{\left(1-k_{2} \phi\right)\left(1+k_{2} \phi\right)}{2 k_{2} M^{2}},
$$

will involve $k_{1}$ or $k_{2}$. Moreover, they will be undefined for the zero limit of these parameters. This completes the first stage extension and with this irreducible as well as covariant set of SCCs (21) we now proceed to the second stage extension in the Batalin-Tyutin formalism [7].

\section{IV: BATALIN-TYUTIN EXTENSION AND NON-COMMUTATIVE SPACE-TIME}

The basic idea behind the BT scheme [7, [6] is to introduce additional phase space variables (BT variables) $\phi_{a}^{\alpha}$, besides the existing degrees of freedom, such that all the constraints in the extended system are converted to FCCs. The advantage is that the FCC system, being a gauge theory, enjoys more freedom in the form of choice of gauge in quantization and the quantization procedure itself is well understood for a gauge theory in a canonical phase space. 
This means that one has to modify the original constraints and Hamiltonian accordingly by putting BT-extension terms in them. The way to achieve this at the classical level has been provided in [7]. The main results of the BT prescription [7] relevant for our purpose are listed below.

Let us consider a set of constraints $\left(\bar{\Theta}_{\alpha}^{\mu}, \Psi_{l}\right)$ and a Hamiltonian operator $H$ with the following $\mathrm{PB}$ relations,

$$
\begin{gathered}
\left\{\bar{\Theta}_{\alpha}^{\mu}(q), \bar{\Theta}_{\beta}^{\nu}(q)\right\} \approx \bar{\Delta}_{\alpha \beta}^{\mu \nu}(q) \neq 0 ; \quad\left\{\bar{\Theta}_{\alpha}^{\mu}(q), \Psi_{l}(q)\right\} \approx 0 \\
\left\{\Psi_{l}(q), \Psi_{n}(q)\right\} \approx 0 ; \quad\left\{\Psi_{l}(q), H(q)\right\} \approx 0 .
\end{gathered}
$$

In the above $(q)$ collectively refers to the set of variables present prior to the BT extension and $" \approx "$ means that the equality holds on the constraint surface. Clearly $\bar{\Theta}_{\alpha}^{\mu}$ and $\Psi_{l}$ are SCC and FCC [5] respectively. These constraints are actually the ones we have been working with, i.e. $\bar{\Theta}_{\alpha}^{\mu}$ are given in (21) and $\Psi_{1}$ of the starting FCCs (9) remains unchanged, whereas $\Psi_{2}$ can be modified to make it an FCC, (at least up to low order in the auxiliary variables). However, this restriction is not important for our present discussion.

In systems with non-linear SCCs, (such as the present one), in general the DBs can become dynamical variable dependent [10, 3] due to the $\left\{A, \bar{\Theta}_{\alpha}^{\mu}\right\}$ and $\bar{\Delta}_{\alpha \beta}^{\mu \nu}$ terms, leading to problems for the quantization programme. To cure this type of pathology, BT formalism is a systematic framework where one introduces the BT variables $\phi_{a}^{\alpha}$, obeying

$$
\left\{\phi_{\mu}^{\alpha}, \phi_{\nu}^{\beta}\right\}=\omega_{\mu \nu}^{\alpha \beta}=-\omega_{\nu \mu}^{\beta \alpha},
$$

where $\omega_{\mu \nu}^{\alpha \beta}$ is a constant (or at most a c-number function) matrix, with the aim of modifying the $\operatorname{SCC} \bar{\Theta}_{\alpha}^{\mu}(q)$ to $\tilde{\Theta}_{\alpha}^{\mu}\left(q, \phi_{\mu}^{\alpha}\right)$ such that,

$$
\left\{\tilde{\Theta}_{\alpha}^{\mu}(q, \phi), \tilde{\Theta}_{\beta}^{\nu}(q, \phi)\right\}=0 ; \tilde{\Theta}_{\alpha}^{\mu}(q, \phi)=\Theta_{\alpha}^{\mu}(q)+\Sigma_{n=1}^{\infty} \tilde{\Theta}_{\alpha}^{\mu(n)}(q, \phi) ; \quad \tilde{\Theta}^{\mu(n)} \approx O\left(\phi^{n}\right)
$$

This means that $\tilde{\Theta}_{\alpha}^{\mu}$ are now FCCs and in particular abelian [7]. A simple choice, obviously not unique, is

$$
\omega_{\mu \nu}^{\alpha \beta}=g_{\mu \nu} \epsilon^{\alpha \beta}, \epsilon^{12}=1 .
$$

The explicit terms in the above expansion are [7],

$$
\begin{gathered}
\tilde{\Theta}_{\alpha}^{\mu(1)}=X_{\alpha \beta}^{\mu \nu} \phi_{\nu}^{\beta} ; \bar{\Delta}_{\alpha \beta}^{\mu \nu}+X_{\alpha \delta}^{\mu \lambda} \omega_{\lambda \sigma}^{\delta \gamma} X_{\beta \gamma}^{\nu \sigma}=0 \\
\tilde{\Theta}_{\alpha}^{\mu(n+1)}=-\frac{1}{n+2} \phi_{\sigma}^{\delta} \omega_{\delta \gamma}^{\sigma \lambda} X_{\lambda \nu}^{\gamma \beta} B_{\beta \alpha}^{\nu \mu(n)} ; n \geq 1 \\
B_{\beta \alpha}^{\nu \mu(1)}=\left\{\tilde{\Theta}_{\beta}^{\nu(0)}, \tilde{\Theta}_{\alpha}^{\mu(1)}\right\}_{(q)}-\left\{\tilde{\Theta}_{\alpha}^{\mu(0)}, \tilde{\Theta}_{\beta}^{\nu(1)}\right\}_{(q)} \\
B_{\beta \alpha}^{\mu \nu(n)}=\Sigma_{m=0}^{n}\left\{\tilde{\Theta}_{\beta}^{\nu(n-m)}, \tilde{\Theta}_{\alpha}^{\mu(m)}\right\}_{(q, p)}+\Sigma_{m=0}^{n}\left\{\tilde{\Theta}_{\beta}^{\nu(n-m)}, \tilde{\Theta}_{\alpha}^{\mu(m+2)}\right\}_{(\phi)} ; n \geq 2
\end{gathered}
$$

In the above, we have defined,

$$
X_{\alpha \gamma}^{\mu \nu} X_{\nu \lambda}^{\gamma \beta}=\omega_{\alpha \gamma}^{\mu \nu} \omega_{\nu \lambda}^{\gamma \beta}=\delta_{\alpha}^{\beta} \delta_{\lambda}^{\mu}
$$


A very useful idea is to introduce the Improved Variable $\tilde{f}(q)$ [] corresponding to each $f(q)$,

$$
\begin{gathered}
\tilde{f}(q, \phi) \equiv f(\tilde{q})=f(q)+\sum_{n=1}^{\infty} \tilde{f}(q, \phi)^{(n)} ; \tilde{f}^{(1)}=-\phi_{\mu}^{\alpha} \omega_{\alpha \beta}^{\mu \nu} X_{\nu \lambda}^{\beta \delta}\left\{\bar{\Theta}_{\delta}^{\lambda}, f\right\}_{(q)} \\
\tilde{f}^{(n+1)}=-\frac{1}{n+1} \phi_{\mu}^{\alpha} \omega_{\alpha \beta}^{\mu \nu} X_{\nu \lambda}^{\beta \delta} G(f)_{\delta}^{\lambda(n)} ; n \geq 1 \\
G(f)_{\beta}^{\mu(n)}=\sum_{m=0}^{n}\left\{\tilde{\Theta}_{\beta}^{\mu(n-m)}, \tilde{f}^{(m)}\right\}_{(q)}+\Sigma_{m=0}^{(n-2)}\left\{\tilde{\Theta}_{\beta}^{\mu(n-m)}, \tilde{f}^{(m+2)}\right\}_{(\phi)}+\left\{\tilde{\Theta}_{\beta}^{\mu(n+1)}, \tilde{f}^{(1)}\right\}_{(\phi)}
\end{gathered}
$$

which have the property $\left\{\tilde{\Theta}_{\alpha}^{\mu}(q, \phi), \tilde{f}(q, \phi)\right\}=0$. Thus the improved variables are FC or equivalently gauge invariant. The subscript $(\phi)$ and $(q)$ in the PBs indicate the variables with respect to which the PBs are to be taken. It can be proved that extensions of the original FCC $\Psi_{l}$ and Hamiltonian $H$ are simply,

$$
\tilde{\Psi}_{l}=\Psi(\tilde{q}) ; \quad \tilde{H}=H(\tilde{q}) .
$$

One can also reexpress the converted SCCs as $\tilde{\Theta}_{\alpha}^{\mu} \equiv \Theta_{\alpha}^{\mu}(\tilde{q})$. The following identification theorem,

$$
\{\tilde{A}, \tilde{B}\}=\{A, \tilde{B}\}_{D B} \quad ;\left.\quad\{\tilde{A}, \tilde{B}\}\right|_{\phi=0}=\{A, B\}_{D B} \quad ; \quad \tilde{0}=0,
$$

plays a crucial role in this scheme in making contact with the DBs. Hence the outcome of the BT extension is the closed system of FCCs with the FC Hamiltonian given below,

$$
\left\{\tilde{\Theta}_{\alpha}^{\mu}, \tilde{\Theta}_{\beta}^{\nu}\right\}=\left\{\tilde{\Theta}_{\alpha}^{\mu}, \tilde{\Psi}_{l}\right\}=\left\{\tilde{\Theta}_{\alpha}^{\mu}, \tilde{H}\right\}=0 ;\left\{\tilde{\Psi}_{l}, \tilde{\Psi}_{n}\right\} \approx 0 ;\left\{\tilde{\Psi}_{l}, \tilde{H}\right\} \approx 0 .
$$

We will see that due to the non-linearity in the SCCs, the extensions in the improved variables, (and subsequently in the FCCs and FC Hamiltonian), turn out to be infinite series. This type of situation has been encountered before [10, 3]. In the present case, the solution for $X_{\alpha \beta}^{\mu \nu}$ in (38) is obtained as,

$$
X_{\alpha \beta}^{\mu \nu}=\left(\begin{array}{cc}
-\bar{\Delta}_{11}^{\mu \nu} & \frac{1}{2} g^{\mu \nu} \\
2 \bar{\Delta}_{12}^{\mu \nu} & 0
\end{array}\right) .
$$

The inverse of the above matrix, as defined in (42), is

$$
X_{\mu \nu}^{\alpha \beta}=\left(\begin{array}{cc}
0 & \frac{1}{2}\left(a_{1} P_{\mu} P_{\nu}+a_{2} g_{\mu \nu}\right) \\
2 g_{\mu \nu} & a_{2}\left(\bar{\Delta}_{11}\right)_{\mu \nu}
\end{array}\right) .
$$

The parameters $a_{1}$ and $a_{2}$ have already been defined in (28). Using (38), the one- $\phi$ BT extensions in the SCCs are,

$$
\begin{gathered}
\tilde{\Theta}_{1}^{\mu(1)}=X_{11}^{\mu \nu} \phi_{\nu}^{1}+X_{12}^{\mu \nu} \phi_{\nu}^{2}=-\bar{\Delta}_{11}^{\mu \nu} \phi_{\nu}^{1}+\frac{1}{2} \phi^{2 \mu}, \\
\tilde{\Theta}_{2}^{\mu(1)}=X_{21}^{\mu \nu} \phi_{\nu}^{1}+X_{22}^{\mu \nu} \phi_{\nu}^{2}=2 \bar{\Delta}_{12}^{\mu \nu} \phi_{\nu}^{1} .
\end{gathered}
$$

It is easy to convince oneself that the $B_{\alpha \beta}^{\mu \nu}$ functions defined in $(39,40,41)$ are in general nonvanishing giving rise to terms having higher powers in BT variables. Let us now compute 
the one- $\phi$ extension in the $\tilde{x}_{\mu}$, i.e. the improved variable corresponding to $x_{\mu}$, the canonical coordinate variable. Simplifying (43), we get,

$$
\begin{gathered}
\tilde{x}_{\mu}^{(1)}=-\left(\phi^{\alpha}\right)^{\nu} \epsilon_{\alpha \beta} X_{\nu \sigma}^{\beta \gamma}\left\{\bar{\Theta}_{\gamma}^{\sigma}, x_{\mu}\right\}_{(q)} \\
=\left[2\left(S_{\nu \mu}+k_{1} \pi g_{\nu \mu}\right)+\frac{1}{M}\left(k_{2} \phi-1\right) a_{2}\left(\bar{\Delta}_{11}\right)_{\nu \mu}\right]\left(\phi^{1}\right)^{\nu} \\
+\frac{1}{2 M}\left(1-k_{2} \phi\right)\left(a_{1} P_{\mu} P_{\nu}+a_{2} g_{\mu \nu}\right)\left(\phi^{2}\right)^{\nu} .
\end{gathered}
$$

Hence, up to one- $\phi_{\mu}^{\alpha}$ BT extension, it is straightforward to check the following relation,

$$
\left\{x^{\mu}+\tilde{x}^{(1) \mu}, x^{\nu}+\tilde{x}^{(1) \nu}\right\}=-\frac{S^{\mu \nu}}{M^{2}}+\left(\phi^{\alpha}-\text { terms }\right) .
$$

In the above calculation, one has to remember that the BT extended expressions for the FCCs has to be used. The PB between the full $\tilde{x}_{\mu}$, (i.e. to all orders in $\phi^{\alpha}$ ), will satisfy

$$
\left\{\tilde{x}^{\mu}, \tilde{x}^{\nu}\right\}=-\frac{\tilde{S}^{\mu \nu}}{M^{2}}
$$

To ascertain the one- $\phi_{\mu}^{\alpha}$ term in the right hand side of the above PB, one has to compute extensions up to two- $\phi_{\mu}^{\alpha}$ in $\tilde{x}_{\mu}$. Thus, we have explicitly derived the following relation,

$$
\tilde{x}_{\mu}=x_{\mu}+\tilde{x}_{\mu}^{(1)}+\left(\text { higher } \phi^{\alpha}-\text { terms }\right)
$$

with $\tilde{x}_{\mu}^{(1)}$ given by (52). This is the cherished mapping between the NC space-time coordinate $\tilde{x}_{\mu}$ and the usual space-time coordinate $x_{\mu}$. A similar type of mapping between an NC coordinate and a canonical coordinate was also proposed, (in a non-relativistic framework), in [14. However, it should be pointed out that $x_{\mu}$ in the above mapping (55) is truly the usual space-time coordinate, with correct Lorentz transformation properties, whereas the canonical coordinate introduced in [14] is not. The BRST quantization of this irreducible SCC system does not pose any technical complications.

The presence of non-commutativity has made a strong impact in recent years in High Energy Physics, ever since the appearance of the seminal work of Seiberg and Witten [15]. Noncommutativity is induced in the open string boundaries, when the string moves in a constant two-form background Neveu-Schwarz field (or equivalently a magnetic field). The noncommutativity parameter is identified with the inverse of the constant magnetic field. Recently we have shown [16] that contrary to previous works [17] involving constraints, the non-trivial mixed boundary conditions are responsible for this noncommutativity. However, the issue of noncommutativity is quite alive and different avenues have to be explored to obtain the above feature. Exploiting the observation that noncommutativity appears in a particular choice of regularization in string theory, Seiberg and Witten [15] have provided an explicit map, (to the lowest nontrivial order in the noncommutativity pa! rameter), connecting noncommutative and ordinary gauge fields. The idea of equivalence between gauge orbits in ordinary and noncommutative space-time plays a pivotal role in extablishing the map. Apparantly, the noncommutativity of space-time is not exploited directly since one stays in ordinary the space-time and introduces extra interaction terms in the original model as noncommutative effects. 
On the other hand, in [18] we have shown a new way of interpreting the Seiberg-Witten map [15] which is more geometric in nature and rests directly on space-time noncommutativity. In [18 the Seiberg-Witten map appears as one changes the argument of the gauge field from ordinary to noncommutative space-time in a particular way. This means that without going in to the concept of identifying gauge equivalence between noncommutative and commutative space-times, it is possible to recover the Seiberg-Witten map in a geometrical way. The spinning particle lives in an extended space having noncommutative and commutative sectors and gauge fields in these sectors can be connected by the Seiberg-Witten map. Precisely in this context the above spinning particle model can become relevant since they provide a natural framework for introducing noncommutativity in space-time, without any need to bring in external interactions. The details of this mechanism will be reported elsewhere.

\section{PROJECTION OPERATOR METHOD}

Recently a new scheme, the Projection Operator Method, has been proposed by Batalin, Lyakhovich and Marnelius [9], where one is able to quantize a constrained system, having a set of reducible SCCs and FCCs, in a manifestly covariant framework. A generalized BRST operator has also been posited in [9]. The formalism is in complete contrast to the Auxiliary Variable approach [8] and BT extension [7] discussed in the previous sections. Here no nonphysical degrees of freedom are introduced. Instead, the major task is the construction of an invariant projection operator that projects out the maximal subset of constraints in involution, (i.e. FCCs), from the full (reducible) set of constraints. With this set of reducible FCCs one can attempt a BRST quantization. However, the presence of SCCs causes an obstruction, which requires a generalization of the BRST operator [9]. In [9] the authors make the crucial assumption that for a (reducible) set of constraints, with the PB algebra,

$$
\left\{\Theta_{\alpha}, \Theta_{\beta}\right\}=U_{\alpha \beta}^{\gamma} \Theta_{\gamma}+\Delta_{\alpha \beta},
$$

one can construct a suitable projection matrix $P_{\alpha}{ }^{\beta}$ satisfying,

$$
P_{\alpha}^{\beta} \Delta_{\beta \gamma} P_{\chi}^{\gamma}=0, P_{\alpha}^{\beta} P_{\beta}^{\gamma}=P_{\alpha}^{\gamma}
$$

This will project out the reducible set of FCCs ,

$$
\Theta_{\alpha}^{\prime}=P_{\alpha}^{\beta} \Theta_{\beta}, \quad\left\{\Theta_{\alpha}^{\prime}, \Theta_{\beta}^{\prime}\right\} \approx 0 .
$$

It is imperative to show that the above assumption works in a non-trivial model and the present work is probably the first one where its validity is demonstrated explicitly. The formalism [9] is applicable even in systems where one can not separate out the FCCs and SCCs without spoiling manifest covariance. However, as shown in section II, in our model this separation is possible. This slightly simplifies the problem since we have to consider only the reducible SCCs $\Theta_{\alpha}^{\mu}$ in (12).

The all important projection operator is given by the following matrix,

$$
P_{\alpha}^{\beta}=\left(\begin{array}{cc}
\frac{1}{M^{2}} S^{\mu \sigma} P_{\sigma} P^{\nu} & S^{\mu \nu} \\
\frac{1}{M^{2}} P^{\mu} P^{\nu} & \left(g^{\mu \nu}+\frac{1}{M^{2}} P^{\mu} S^{\nu \sigma} P_{\sigma}\right)
\end{array}\right) .
$$


This will lead to the FCCs $\Theta_{\alpha}^{\prime}=P_{\alpha}^{\beta} \Theta_{\beta}$ whose closure property can be directly checked. Now one must enlarge the phase space by introducing ghosts, ghosts for ghosts etc. We follow the prescription of [9] and for a generic $L$ 'th stage reducible theory introduce the ghosts $\mathcal{C}^{a}, \overline{\mathcal{P}}_{a}$ and secondary ghosts $\mathcal{C}^{\prime a_{r}}, \overline{\mathcal{P}}_{a_{r}}^{\prime}$ where $r=1, . ., L$ and the ghosts satisfy

$$
\begin{gathered}
\epsilon\left(\mathcal{C}^{a}\right)=\epsilon\left(\overline{\mathcal{P}}_{a}\right)=\epsilon_{a}+1, \epsilon\left(\mathcal{C}^{\prime a_{r}}\right)=\epsilon\left(\overline{\mathcal{P}}_{a_{r}}^{\prime}\right)=\epsilon_{a_{r}}+r+1, r=1, . ., L, \\
\left\{\mathcal{C}^{a}, \overline{\mathcal{P}}_{b}\right\}=\delta_{b}^{a},\left\{\mathcal{C}^{\prime a_{r}}, \overline{\mathcal{P}}_{b_{r}}^{\prime}\right\}=\delta_{b_{r}}^{a_{r}}, r=1, . ., L, \\
g h\left(\mathcal{C}^{a}\right)=-g h\left(\overline{\mathcal{P}}_{a}\right)=1, g h\left(\mathcal{C}^{\prime a_{r}}\right)=-g h\left(\overline{\mathcal{P}}_{a_{r}}^{\prime}\right)=r+1 .
\end{gathered}
$$

Here $\epsilon(\mathcal{A})$ and $g h(\mathcal{A})$ denote the parity and ghost number of $\mathcal{A}$ and in the above $\epsilon\left(T_{a}\right) \equiv \epsilon_{a}$, where $T_{a}$ represents the full set of constraints. Let us now construct the following odd real function $\Omega$ with ghost number 1 ,

$$
\begin{gathered}
\Omega=\mathcal{C}^{a} T_{a}+\mathcal{C}^{\prime a_{1}} Z_{a_{1}}^{a} \overline{\mathcal{P}}_{a}(-1)^{\epsilon_{a}}+\sum_{r=2}^{L} \mathcal{C}^{a_{r}} Z_{a_{r}}^{a_{r-1}} \overline{\mathcal{P}}_{a_{r-1}}(-1)^{\epsilon_{a_{r-1}}} \\
+(-1)^{\epsilon_{b}} \frac{1}{2} \mathcal{C}^{b} \mathcal{C}^{a} \Delta_{a b}^{e} \overline{\mathcal{P}}_{e}(-1)^{\epsilon_{e}}+(-1)^{\epsilon_{a}} \mathcal{C}^{a} \mathcal{C}^{b^{b_{1}}} \Delta_{b_{1} a}^{a_{1}} \overline{\mathcal{P}}^{\prime}{ }_{a_{1}}(-1)^{\epsilon_{a_{1}}} \\
+(-1)^{\left(\epsilon_{b}+\epsilon_{a} \epsilon_{e}\right)} \frac{1}{6} \mathcal{C}^{e} \mathcal{C}^{b} \mathcal{C}^{a} \Delta_{a b e}^{a_{1}} \overline{\mathcal{P}}^{\prime}{ }_{a_{1}}(-1)^{\epsilon_{a_{1}}}+\ldots
\end{gathered}
$$

In the above we have defined the constraint algebra as,

$$
\left\{T_{a}, T_{b}\right\}=C_{a b}+\Delta_{a b}^{e} T_{e}
$$

and the reducibility conditions as,

$$
Z_{a_{r}}^{a} T_{a}=0, r=1, . . L .
$$

The higher order reducibility conditions are of the form,

$$
Z_{a_{2}}^{a_{1}} Z_{a_{1}}^{a}=0
$$

and so on. The higher order structure function $\Delta_{b_{r} a}^{a_{r}}$ follows from the requirement

$$
\left\{T_{a}, Z_{a_{r}}^{b} T_{b}\right\}=0
$$

wheras $\Delta_{a b e}^{a_{1}}$ is induced by the Jacobi identity

$$
J\left(T_{a}, T_{b}, T_{e}\right) \equiv\left\{\left\{T_{a}, T_{b}\right\}, T_{e}\right\}+\text { cyclic terms }=0 .
$$

Rest of the structure functions will follow from still higher order Poisson Brackets. For a detailed discussion see for example [19].

In the present case, $T_{a} \equiv\left(\Psi_{1}, \Psi_{2}, \Theta_{1}^{\mu}, \Theta_{2}^{\nu}\right)$ in (11, 12) and our model is single stage reducible with $L=1$ (18). One can ascertain that $\Delta_{b_{1} a}^{a_{1}}=0$ from $\left\{T_{a}, P^{\mu} \Theta_{1 \mu}\right\}=0$. $\Delta_{a b e}^{a_{1}}$ contributes only from the non-trivial Jacobi identity expression,

$$
J\left(\Theta_{1}^{\mu}, \Theta_{1}^{\nu}, \Theta_{2}^{\sigma}\right)=\frac{1}{M}\left(g^{\sigma \nu} P^{\mu}-g^{\sigma \mu} P^{\nu}\right) \Psi_{1}+\left(g^{\sigma \nu} \Theta_{2}^{\mu}-g^{\sigma \mu} \Theta_{2}^{\nu}\right) \Psi_{1} .
$$


We drop the second term since it is quadratic in the constraints and hence is strongly vanishing. The rest of the higher order structure functions are zero. With the above inputs, the function $\Omega$ is

$$
\begin{gathered}
\Omega=\mathcal{C}^{1} \Psi_{1}+\mathcal{C}^{2} \Psi_{2}+\mathcal{C}^{1 \mu} \Theta_{1 \mu}+\mathcal{C}^{2 \mu} \Theta_{2 \mu} \\
\left.+\mathcal{C}^{\prime} P^{\mu} \overline{\mathcal{P}}_{1 \mu}+\frac{1}{2} \mathcal{C}^{2 \mu} \mathcal{C}^{1 \nu}\left(\left(g_{\mu \nu} P_{\sigma}-g_{\mu \sigma} P_{\nu}\right) \overline{\mathcal{P}}_{2}^{\sigma}+\frac{1}{M} g_{\mu \nu} \overline{\mathcal{P}}_{1}\right)\right)+\frac{1}{2} \mathcal{C}^{1 \mu} \mathcal{C}^{1 \nu} S_{\mu \nu} \overline{\mathcal{P}}_{1} \\
+\mathcal{C}^{1 \mu} P_{\mu} \mathcal{C}^{1 \nu} \overline{\mathcal{P}}_{1 \nu}+\frac{1}{6 M} \mathcal{C}^{2 \mu} \mathcal{C}_{1 \mu} \mathcal{C}^{1 \nu} P_{\nu} \overline{\mathcal{P}}^{\prime}
\end{gathered}
$$

Next an even real function $\Pi$ with ghost number zero is posited to be

$$
\Pi=\mathcal{C}^{a} P_{a}^{b} \overline{\mathcal{P}}_{b}(-1)^{\epsilon_{b}}+\sum_{r=1}^{L}(-1)^{r} \mathcal{C}^{a_{r}} P_{a_{r}}^{b_{r}} \overline{\mathcal{P}}_{b_{r}}(-1)^{\epsilon_{b_{r}}}+\ldots
$$

where the dots indicate higher powers of ghost terms. The functions $P_{a_{r}}^{\prime b_{r}}$ are yet to be determined from the following relations [9],

$$
\{\Pi,\{\Pi, \Omega\}\}=\{\Pi, \Omega\},\{\Pi,\{\Pi,\{\Omega, \Omega\}\}\}=\{\Pi,\{\Omega, \Omega\}\} .
$$

This leads to the cherished form of the generalized BRST charge

$$
\Omega^{\prime} \equiv\{\Pi, \Omega\}
$$

which obeys the nilpotency property,

$$
\left\{\Omega^{\prime}, \Omega^{\prime}\right\}=0
$$

In the present case, using (59), we get,

$$
\begin{gathered}
\Pi=\mathcal{C}^{1} \overline{\mathcal{P}}_{1}+\mathcal{C}^{2} \overline{\mathcal{P}}_{2}+\frac{1}{M^{2}} \mathcal{C}^{1 \mu} S_{\mu \sigma} P^{\sigma} P^{\nu} \overline{\mathcal{P}}_{1 \nu} \\
+\mathcal{C}^{1 \mu} S_{\mu \nu} \overline{\mathcal{P}}_{2}^{\nu}+\frac{1}{M^{2}} \mathcal{C}^{2 \mu} P_{\mu} P^{\nu} \overline{\mathcal{P}}_{1 \nu}+\frac{1}{M^{2}} \mathcal{C}^{2 \mu} P_{\mu} S^{\nu \sigma} P_{\sigma} \overline{\mathcal{P}}_{2 \nu}-\mathcal{C}^{\prime} P^{\prime} \overline{\mathcal{P}}^{\prime}
\end{gathered}
$$

Computation of the last term is straightforward but tedious and is not pursued here. Just as in the irreducible case, in general one has to modify the Hamiltonian so that it has vanishing PB with the BRST charge $\Omega^{\prime}$. However, in the present case the Hamiltonian remains the same as in $(20)$ due to its simple structure. In the subsequent quantization these functions are elevated to quantum operators and obviously they have to be properly ordered.

\section{CONCLUSIONS}

The covariant quantization of the Nambu-Goto spinning particle model is analyzed and the relevance of this model in inducing a non-commutative space-time is demonstrated. The technical problem of covariant quantization in the present model is very subtle since the set of constraints comprise reducible Second Class Constraints apart from First Class Constraints. (The above classification follows from the prescription of Dirac [5].) Special methods have been devised to tackle the above mentioned reducibility problem. We have discussed here two schemes: (i) the 
auxiliary variable method [8] where the phase space is enlarged in an appropriate way and (ii) the projection operator method [9], where a (reducible) set of first class constraints is projected out from the set of second class constraints. The latter formalism has been proposed very recently and the present work constitutes a non-trivial application of the same. Construction of the projection operator as well as the necessary steps for the BRST quantization has been provided.

A number of projects to be pursued further immediately comes to mind: Firstly a thorough appraisal of the mapping between non-commuting and ordinary space-time that has been exhibited here, in the light of [18], and secondly a quantum BRST analysis of the model in the projection operator formalism taking into account the operator ordering problems. Also it has been suggested [20] that the Faddeev-Jackiw [21] method of symplectic quantization may be useful in the context of covariant quantization of the spinning particle model. Work is in progress in this direction as well.

Acknowledgements: It is a pleasure to thank Professor R.Banerjee and Professor R.Jackiw for helpful comments. 


\section{References}

[1] For a review see M.R.Douglas and N.A.Nekrasov, arXiv: HEP-TH/ 0106048.

[2] For a review see A.Connes, Non-Commutative Geometry, Academic Press, 1994.

[3] S.Ghosh, J.Math.Phys. 42 5202(2001).

[4] A.J.Hanson and T.Regge, Ann.Phys. (N.Y.) 87 498(1974); see also A.J.Hanson, T.Regge and C.Teitelboim, Constrained Hamiltonian System, Roma, Accademia Nazionale Dei Lincei, (1976).

[5] P.A.M.Dirac, Lectures on Quantum Mechanics (Yeshiva University Press, New York, 1964).

[6] E.S.Fradkin and G.A.Vilkovisky, Phys.Lett. B55 224(1975); I.A.Batalin and E.S.Fradkin, Nucl.Phys. B279 514(1987); I.A.Batalin, E.S.Fradkin and T.E.Fradkina, Nucl.Phys. B314 158(1989).

[7] I.A.Batalin and I.V.Tyutin, Int.J.Mod.Phys. A6 3255(1991).

[8] R.Banerjee and J.Barcelos-Neto, Ann.Phys. 265 134(1998).

[9] I.A.Batalin, S.L.Lyakhovich and R.Marnelius, Phys.Lett. B534 201(2002) (arXiv: hepth/0112175).

[10] See for example N.Banerjee, R.Banerjee and S.Ghosh, Nucl.Phys. B417 257(1994); Phys.Rev. D49 1996(1994); Ann.Phys. (N.Y.) 241 237(1995); M.-U. Park and Y.-J. Park, Int.J.Mod.Phys. A13 2179(1998).

[11] S.Ghosh, Phys.Lett. B338 235(1994); Phys.Rev. D51 5827 (1995); J.Phys. A30 L821(1997).

[12] For a review on anyon, see for example F.Wilczek, Fractional Statistics and Anyon Superconductivity (World Scientific, Singapore, 1990).

[13] For a discussion on this point, see M.Henneaux and C.Teitelboim, Quantizations of Gauge Systems, Princeton University Press, Princeton, (1992).

[14] C.Chou, V.P.Nair and A.P.Polychronakos, Phys.Lett. B304 105(1993).

[15] N.Seiberg and E.Witten, JHEP 9909(1999)032 (hep-th/9908142).

[16] R.Banerjee, B.Chakraborty and S.Ghosh, Noncommutativity in open string: New results in a gauge independent analysis, hep-th/0203199, (to appear in Phys.Lett.B).

[17] F.Ardalan, H.Arfaei and M.M.Sheikh-Jabbari, JHEP 9902(1999)016; C.-S.Chu and P.M.Ho, Nucl.Phys. B550 151(1999).

[18] S.Ghosh, A new interpretation of the Seiberg-Witten Map? , hep-th/0203143.

[19] R.Ferraro, M.Henneaux and M.Puchin, J.Math.Phys. 34 2757(1993). 
[20] R.Jackiw, (private communication).

[21] L.Faddeev and R.Jackiw, Phys.Rev.Lett. 60 1692(1988). 